\title{
A Survey on Software Defect Prediction Using Deep Learning
}

\author{
Elena N. Akimova 1,2,*(D), Alexander Yu. Bersenev ${ }^{1,2}$, Artem A. Deikov ${ }^{1,2}$, Konstantin S. Kobylkin 1,2, \\ Anton V. Konygin ${ }^{1}$, Ilya P. Mezentsev ${ }^{1,2}$ and Vladimir E. Misilov ${ }^{1,2}$ (D) \\ 1 Krasovskii Institute of Mathematics and Mechanics, Ural Branch of RAS, S. Kovalevskaya Street 16, \\ 620108 Ekaterinburg, Russia; Alexander.Bersenev@urfu.ru (A.Yu.B.); deykov.artem@urfu.ru (A.A.D.); \\ kobylkin@imm.uran.ru (K.S.K.); konygin@imm.uran.ru (A.V.K.); ilya.mezentsev@urfu.ru (I.P.M.); \\ v.e.misilov@urfu.ru (V.E.M.) \\ 2 Institute of Radioelectronics and Information Technology, Ural Federal University, Mira Street 19, \\ 620002 Ekaterinburg, Russia \\ * Correspondence: aen@imm.uran.ru
}

check for

updates

Citation: Akimova, E.N.;

Bersenev, A.Yu.; Deikov, A.A.;

Kobylkin, K.S.; Konygin, A.V.;

Mezentsev, I.P.; Misilov, V.E. A Survey

on Software Defect Prediction Using

Deep Learning. Mathematics 2021, 9 ,

1180. https://doi.org/10.3390/

math9111180

Academic Editors: Theodore E. Simos and Charampos Tsitouras

Received: 27 April 2021

Accepted: 18 May 2021

Published: 24 May 2021

Publisher's Note: MDPI stays neutral with regard to jurisdictional claims in published maps and institutional affiliations.

Copyright: (C) 2021 by the authors. Licensee MDPI, Basel, Switzerland. This article is an open access article distributed under the terms and conditions of the Creative Commons Attribution (CC BY) license (https:/ / creativecommons.org/licenses/by/ $4.0 /)$.

\begin{abstract}
Defect prediction is one of the key challenges in software development and programming language research for improving software quality and reliability. The problem in this area is to properly identify the defective source code with high accuracy. Developing a fault prediction model is a challenging problem, and many approaches have been proposed throughout history. The recent breakthrough in machine learning technologies, especially the development of deep learning techniques, has led to many problems being solved by these methods. Our survey focuses on the deep learning techniques for defect prediction. We analyse the recent works on the topic, study the methods for automatic learning of the semantic and structural features from the code, discuss the open problems and present the recent trends in the field.
\end{abstract}

Keywords: defect prediction; anomaly detection; program analysis; code understanding; neural networks; deep learning

MSC: 68N30; 68T07

\section{Introduction}

According to the IEEE Standard Classification for Software Anomalies [1], a software defect is "an imperfection or deficiency in a work product where that work product does not meet its requirements or specifications and needs to be either repaired or replaced".

Software defects can cause different problems. Common ways to find software defects are manual testing and code review. The main drawback of these methods is that they are quite expensive in terms of time and effort. The automatic approaches to the Software Defect Prediction (SDP) would allow one to reduce the costs and improve quality of the software projects.

Thus, Software Defect Prediction is an important problem in the fields of the software engineering and programming language research. The task is to identify the defective code with high accuracy (in terms of the precision and recall).

The development and breakthrough of machine learning led to the fact that many tasks can be solved by the these methods.

Recent advances in the fields of artificial neural networks and machine learning, as well as the increasing power of the modern computers (such as supercomputers based on GPUs with AI accelerating modules), allowed new concepts, such as deep learning, to emerge. The main idea is that an artificial neural network with multiple layers is capable of progressively extracting the higher-level features from the original data to solve complex problems.

For the problem of software defect prediction, the researchers have proposed the representation-learning algorithms to learn semantic representations of programs automatically 
and use this representation to identify the defect-prone code. Using these implicit features shows better results than the previous approaches based on the explicit features, such as the code metrics [2].

The software defect prediction is a rapidly developing field, and the state-of-the-art surveys on the topic [3-5] do not sufficiently cover the recent works describing the cuttingedge techniques. For example, recent advances in the related fields of Natural Language Processing (NLP) provided new powerful tools such as Transformer language models. These techniques were later successfully applied to the software engineering tasks.

The goal of our survey is to describe these latest achievements taking into account the newest primary studies published in 2019-2021. We hope that this survey can be useful for researchers and practitioners in the software defect prediction, code understanding and other related fields.

Some semantic defects are hard to find using only source code. For example, in [6], the bytecode of Kotlin programs is processed to detect the so called compiler-induced anomalies, which arise only in the compiled bytecode. Another example is presented in [7] where to expose the program behavior, the assembly code (generated from the C source code by the compiler) is used to learn the defect features.

Nevertheless, the source code remains the main source of data for the defect prediction. In this survey, our main interest lies in techniques devoted to analyzing the source code. Usually, the process of developing the model for the defect prediction consists of the following steps (see Figure 1):

1. Prepare the dataset by collecting the source code samples from repositories of the software projects (or choose the suitable existing dataset).

2. Extract features from the source code.

3. Train the model using the train dataset.

4. Test the model using the test dataset and assess the performance using the quality metrics.

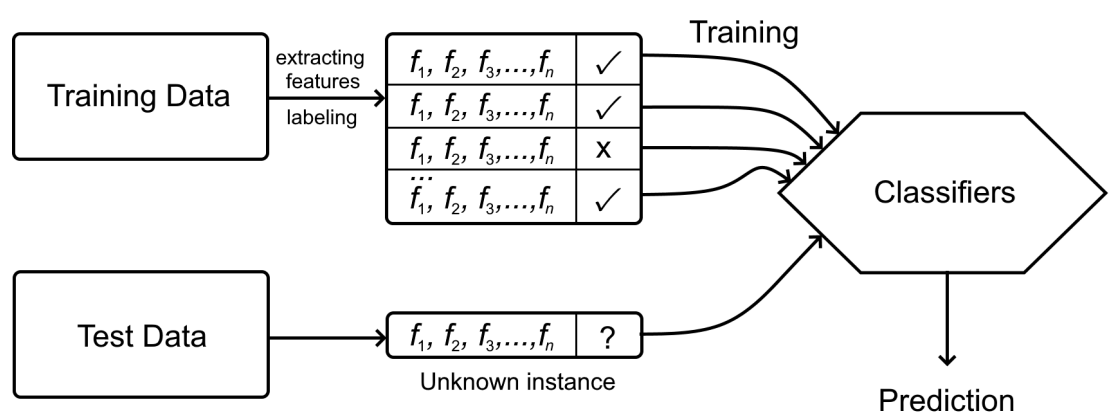

Figure 1. Scheme of the the process of constructing the defect prediction model.

The survey is structured as follows: Section 2 briefly describes the methodology of our survey. Section 3 presents the overview on the various deep learning techniques applied to the defect prediction. In Section 4, we outline the main difficulties of the problem. Section 5 presents the study of the latest trends in the techniques and methods for defect prediction. Section 6 concludes the study and offers our vision on the future developments on the field.

\section{Methodology}

We reviewed the primary studies on the subject. In this section, we present details of our methodology.

\subsection{Research Questions}

To summarize the work of our survey, let us formulate the following research questions: 
- RQ1. What deep learning techniques have been applied to software defect prediction?

- RQ2. What are the key factors contributing to the difficulty of the problem?

- RQ3. What are the trends in the primary studies on the use of deep learning for the software defect prediction?

\subsection{Literature Search and Inclusion or Exclusion Criteria}

To collect related papers, we formulated a search string for Google Scholar and Scopus combining the related keywords "software engineering", "deep learning", and "defect prediction".

To filter the papers with insufficient content and determine the paper quality, we used the following criteria:

- The paper must describe a technique for automatic feature extraction using deep learning and apply it to the defect prediction problem.

- The paper length must not be less than six pages.

\section{RQ1. What Techniques Have Been Applied to This Problem?}

In order to work with the source code, we need to have its representation. On the one hand, this representation should be simple as a vector, since most machine learning algorithms work with vectors. On the other hand, the representation should contain all the necessary information. The numerical vector representing the source code is called an "embedding".

There are different ways to represent the source code. Moreover, we need different granularities for different tasks, for example, for code completion we need token-level embedding and for function clone detection we need function embedding. For the software defect prediction problem, various levels of granularity are used, such as sub-system, component, file/class, method and change (see [8,9] for more info on various code embeddings).

One way is to create the vector from the hand crafted features. This approach assumes that an expert invents a set of features and selects best of them (e.g., $[10,11])$. Usually, these features include the statistical characteristics of code, such as its size, code complexity, code churn or process metrics.

Another way is to create the numerical vector by processing the source code.

One way to represent the code is a sequence of elements. Usually, they are code tokens or characters [12]. The neural networks based on the sequences are usually trained to predict the subsequent element.

Another approach to build the representation of the source code is the abstract syntax trees (AST) [13]. The nodes of the tree correspond to the statement and operators, and the leaves represent the operands and values. The tree-based models are trained to predict the code by generating new nodes taking into account the existing tree structure.

The most common approach to defect prediction is to use some classification algorithm to divide the source code into two categories: defect code and correct one (e.g., [14]).

However, the approaches based on the hand-crafted features usually do not sufficiently capture the syntax and semantics of the source code. Most traditional code metrics cannot distinguish code fragments if these fragments have the same structure and complexity but implement a different functionality. For example, if we switch several lines in the code fragments, traditional features, such as the number of lines of code, number of function calls and number of tokens, would remain the same (see [2]). Thus, the semantic information is more important for defect prediction than these metrics.

Modern approaches are usually based on extracting the implicit structural, syntax and semantic feature from the source code rather than using the explicit hand-crafted ones.

The most popular deep learning techniques for software defect prediction are: Deep Belief Networks (DBN), Convolutional Neural Networks (CNN), Long Short Term Memory (LSTM), and Transformer architecture. 


\subsection{Deep Belief Networks}

Deep Belief Network [15] generative models are based on a multilevel neural network. This network contains one input layer, one output layer and multiple hidden layers. The output layer generates a feature vector representing the data fed to the input layer. Each layer consists of the stochastic nodes. The important feature of the DBN is that the nodes are only connected to the nodes in the adjacent layers but not to the nodes within the same layer as shown in Figure 2.

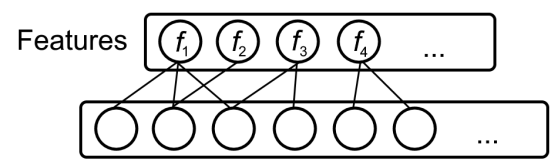

Output Layer

Hidden Layers

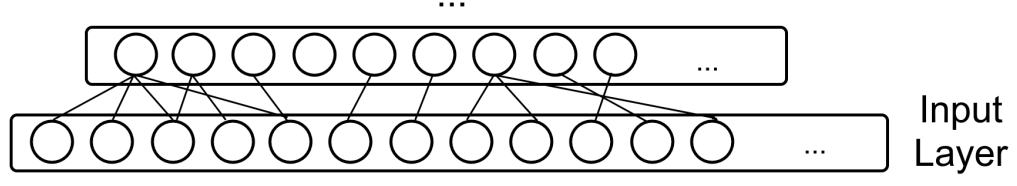

Figure 2. Architecture of the Deep Belief Network.

Perhaps one of the first works combining AST with the deep learning is [16]. The authors propose the approach for software defect prediction on a changes level. The DBN (which is fed by the traditional code metrics) generates the new expressive features and use them in classical machine learning classifiers. They extract the relations from the traditional code metrics, such as number of modified modules, directories and files, added and deleted lines, and several features related to the developer's experience. Later, the authors proposed the "TLEL" approach [17] based on the decision tree and ensemble learning for classification.

The works of Wang et al. [2,18] also use the DBN, but in a different manner. For predicting the defects on the basis of the code semantics, the authors have developed a DBN to automatically learn a semantic features from the source code. As the input for the network, the programs' AST and source code changes are used for the cases of file-level and change-level prediction, respectively. Then, the authors use the classical machine learning classifiers and extracted features to classify source code files whether they are buggy or clean.

The main drawback of the DBN is that it does not sufficiently capture the context of the code elements, such as the order of statement execution and function calls.

\subsection{Long Short Term Memory}

The Long Short Term Memory [19] is a subtype of the recurrent neural network specialized for processing the data sequences. The LSTM network consists of LSTM units (see Figure 3). The key element of the unit is a memory cell, which allows the unit to store the values for a short, as well as, for a long time intervals. This provides the LSTM-based models the ability to capture the long-range context information from the source code.

The LSTM-based model was used in work [11] for learning both the semantic and syntactic features of code. The proposed approach represents the code as a sequence of code tokens, which is fed into a LSTM system to transform code into a feature vector and a token state representing the semantic information of the token. Later the Tree-LSTM model was developed using the AST representation as input [20].

A neural bug finding technique is proposed in [21]. The authors train a neural network on examples of the defective and correct code, and then use the resulting binary classifier for bug detection. To prepare a labeled dataset, the authors use the existing static bug detection software to identify the specific kind of bugs. The code is represented as a tokens sequence and converted to a real-value vector by using the one-hot encoding for each token. Then, a bi-directional network with LSTM is used as model. 


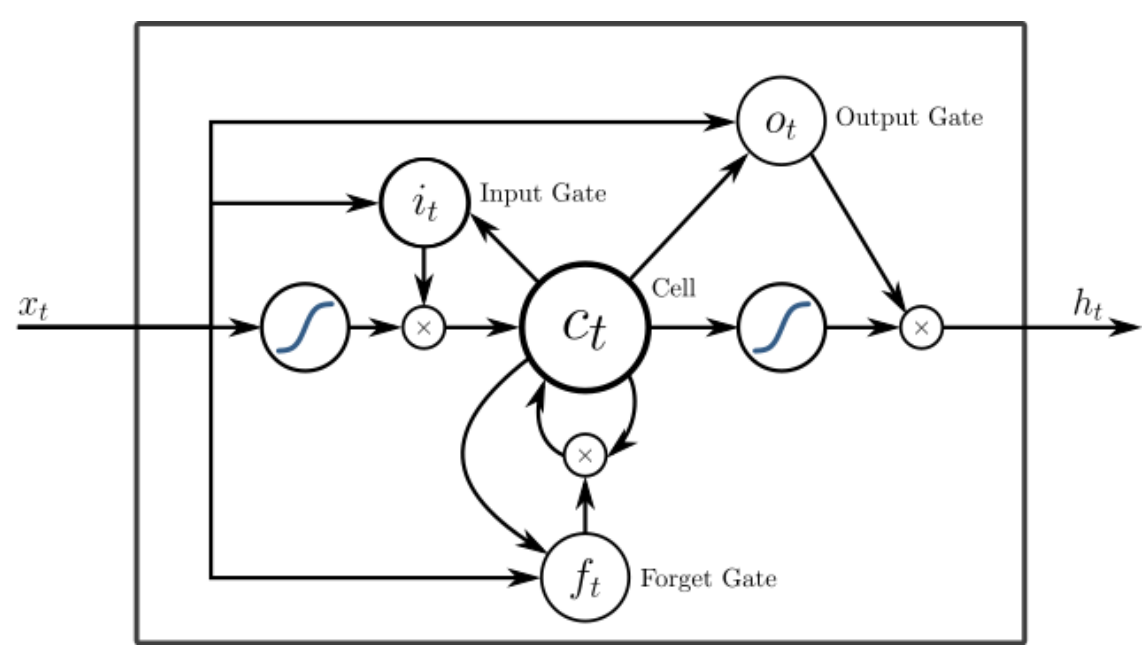

Figure 3. Scheme of the LSTM unit.

In [22], the authors propose a model for defect prediction on the base of AST path pair representation. To process the code, the path in the AST is extracted as combination of symbol sequence and control sequence. These sequences are fed to a Bi-LSTM network to generate a path vector. Then, all the vectors are combined using the global attention technique to generate the vector for the entire code fragment. These final embedding representations are used for classification.

\subsection{Convolutional Neural Networks}

The Convolutional Neural Networks [23] are a type of neural network specialized for processing the data with a mesh-like structure. This network is characterized by two important features. Firstly, the local connection pattern between the units is repeated over the entire network. It allows the network to capture the short-term structural context of the source code. Secondly, the each unit have the same parameters. It allows the network to learn the information on the code element irrespective of its position in the code. The scheme of general CNN is shown in Figure 4.

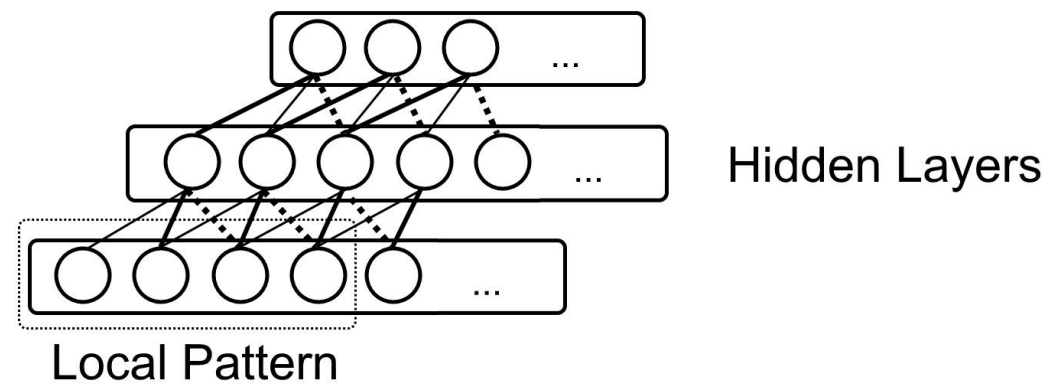

Figure 4. Architecture of the Convolutional Neural Network.

Reference [24] presents the model based on the CNN architecture. Based on the program's AST, the token vectors are extracted and converted to numerical vectors. Then, these vectors are fed into a CNN. After that, the combination of the extracted semantic and structural features and code metrics is used for software defect prediction applying the logistic regression.

A deep learning model to predict defects on the basis of the commit messages and code changes is developed in [25]. This model is based on the CNN. It uses the convolutional network layers for processing the code changes and commit text and the feature combination layer to fuse these two embedding vectors into a single one.

Another deep learning-based model for defect prediction is proposed in [26]. The training of the neural network utilizes the triplet loss technique and the weighted cross-entropy loss technique. The random forest is used as a classifier. 
In [27], the features learning technique based on $\mathrm{CNN}$ is proposed. This model extract features from token vectors in the AST of the code and learns the transferable joint features. Combining these deep-learning-generated features with the hand-crafted ones allows the model to perform the cross-project defect prediction. Later, the authors propose a new treebased convolutional network to perform this task [28]. It uses the tree-based continuous bag-of-word for encoding the AST nodes to be fed into CNN.

\subsection{Transformer Models}

Recently, the big success of pre-trained contextual representations in the NLP, for example, [29], led to a rise of attempts to apply these techniques to source code. Usually, these models are based on the multi-layer Transformer architecture [30] shown in Figure 5. They are pre-trained using the massive unlabeled corpora of programs with the self-supervised objectives, such as masking language modeling and next sentence prediction [31,32]. After the pre-training phase, the model can be fine-tuned for specific tasks using the supervised techniques.

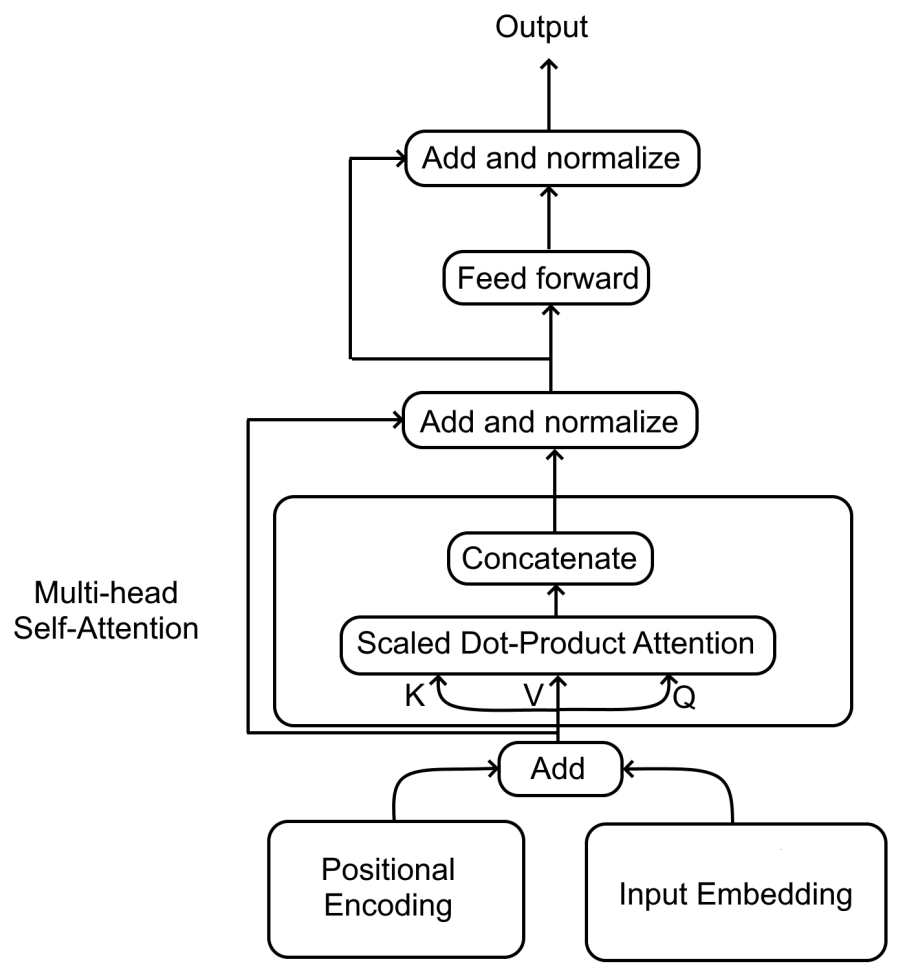

Figure 5. Architecture of the multi-layer transformer.

The authors of [33] state that the approaches based on the traditional complexity metrics are useless since there is no need for a tool to tell the engineer that longer and more complex code is more defect-prone. The methods of learning features from the source code do not guarantee capturing semantic and syntactical similarity, and very similar source codes can have very different features. These features can correlate with defects rather than directly cause them. In contrast, the authors propose an approach based on the self attention transformer encoder to the semantic defect prediction. The matrix representing the defectiveness of each token in the fragment is generated. Attention and layer normalization are used as a regularization technique. The resulting model provides the defect prediction with the semantic highlight of defective code regions.

The CuBERT model is presented in [31]. The authors use a corpus of Python files from the GitHub to create a benchmark for evaluating code embeddings on five classification tasks and a program repair task. They train their model and compare it with various other 
models including the BiLSTM and Transformer. It is shown that the CuBERT outperforms the baseline models consistently.

A bimodal language model called CodeBERT is presented in [32]. It is based on the multilayer bidirectional Transformer neural architecture. To prepare the data, the natural language text is represented as a sequence of words, and the source code is presented as a sequence of tokens. The output of the CodeBERT model is a contextual vector learned from the natural language and source code, as well as the aggregated sequence. The resulting model efficiently solves the problems of both code to the documentation and natural language code search.

Work [34] presents a multi-layer bidirectional transformer architecture GraphCodeBERT, which utilizes three components as input: the source code, paired comments and data flow graph. Data flow graph represents relations between variables, for example, where the value of a variable comes from. This allows the model to consider the code structure for code representation. For pre-training tasks, the traditional masked language modeling, as well as the edge prediction and node alignment of data flow graph were used. It supports several downstream code-related tasks including the code clone detection, code translation and code refinement.

\subsection{Other Networks}

In [35], a software defect prediction technique based on stacked denoising autoencoders model is presented. The stacked denoising autoencoder is used to extract higherlevel features from the traditional metrics. The two-stage ensemble learning is used for classification. To address the class imbalance, the authors use the ensemble learning strategy. Later, the feature selection algorithm was applied to this method to address the feature redundancy problem [36].

A model for the software defect prediction was constructed in work [37] on the base of the Siamese parallel fully-connected networks. This model utilizes the paired parallel Siamese networks architecture and the deep learning approach. The network produces the high-level features that are used for classification. To address the imbalance between the minority and majority classes, the network takes into account the cost-sensitivity features.

The neural forest networks are used to learn feature representations in [38]. To perform a classification, a decision forest is used. It also guides the learning of the neural network. In [39], a new deep forest model is proposed for the software defect prediction. To detect the essential defect features, it uses the cascade learning strategy, which consists in reforming a set of the random forest classifiers into a layered network.

The graph neural network to predict the software defects is constructed in work [40]. It extracts the semantics and context features from the AST of the code fragments. To capture the defect-related information from the source code, the ASTs for the buggy and fixed version of a fragment are constructed and pruned using the community detection algorithm, which extracts the defect-related subtree. Then, the Graph Neural Network is used to capture the latent defect information.

\section{RQ2. What Are the Key Factors Contributing to Difficulty of the Problem?}

The problem of software defect prediction is considered very complex and very challenging for the machine learning models based on the neural networks.

\subsection{Lack of Data}

One of the difficulties is lack of available large labeled datasets devoted to the defect prediction. To alleviate this problem, one can utilize the pre-trained contextual embeddings. This technique consists in pre-training the language model on a massive corpora of unlabeled source code using the self-supervised objectives, such as masked language modeling, next sentence prediction and replaced token detection.

Table 1 presents the popular unlabeled code datasets suitable for this task. 
Table 1. List of unlabeled datasets.

\begin{tabular}{|c|c|c|c|}
\hline Dataset & Content & Size & Used in Tasks \\
\hline $\begin{array}{l}\text { Bigquery github } \\
\text { repos [31] }\end{array}$ & Python source code & $4 \mathrm{M}$ files & Pre-training CuBERT model \\
\hline Py150 [41] & Python source code, AST & 8423 repos, 149,993 files & Fine-tuning CuBERT model \\
\hline Js150 [42] & Javascript source code, AST & 150,000 source files & $\begin{array}{l}\text { Code Summarization; } \\
\text { Defect Prediction }\end{array}$ \\
\hline Datasets for [43] & Java source code & $\begin{array}{l}9500 \text { projects, } 16 \mathrm{M} \text { samples in the largest } \\
\text { one }\end{array}$ & Code summarization \\
\hline GitHub Java Corpus [44] & Java source code & 11,000 projects & Language Modeling \\
\hline CodeNN Dataset [45] & C\# source code and summaries & 66,015 fragments & Code Captioning \\
\hline Dataset for [6] & $\begin{array}{l}\text { Kotlin source code, AST, } \\
\text { bytecode }\end{array}$ & $\begin{array}{l}47,751 \text { repos, } 932,548 \text { files, 4,044,790 } \\
\text { functions }\end{array}$ & Anomaly detection, defect prediction \\
\hline Dataset for [46] & C\# source code & 29 projects, $2.9 \mathrm{M}$ lines of code & Variable Misuse detection \\
\hline
\end{tabular}

The pre-trained model may then be fine-tuned for the defect prediction using much smaller labeled datasets. Table 2 presents a list of publicly available datasets devoted to the defect prediction. Usually, such datasets include pairs of correct and defective code fragments.

Table 2. List of labeled datasets.

\begin{tabular}{|c|c|c|c|}
\hline Dataset & Content & Size & Used in Tasks \\
\hline $\begin{array}{c}\text { SEIP Lab Software } \\
\text { Defect Prediction } \\
\text { Data [47] }\end{array}$ & Complexity metrics & $\begin{array}{l}5 \text { subsequent releases of } 3 \text { projects from the Java } \\
\text { Eclipse community }\end{array}$ & $\begin{array}{l}\text { Data collection } \\
\text { and linking }\end{array}$ \\
\hline $\begin{array}{l}\text { PROMISE Software } \\
\text { Engineering } \\
\text { Repository [48] }\end{array}$ & $\begin{array}{l}\text { Numeric metrics; reported defects } \\
\text { (false/true) }\end{array}$ & 15,000 modules & Defect prediction \\
\hline $\begin{array}{l}\text { NASA Defect } \\
\text { Dataset [49] }\end{array}$ & $\begin{array}{l}\text { Numeric metrics; reported defects } \\
\text { (false/true) }\end{array}$ & 51,000 modules & Defect prediction \\
\hline REPD datasets [50] & $\begin{array}{l}\text { Numeric metrics, semantic features, } \\
\text { reported defects }\end{array}$ & 10,885 fragments in the largest one & Defect prediction \\
\hline GPHR [40] & Java code and metrics & $\begin{array}{l}3526 \text { pairs of fragments, buggy and fixed, code } \\
\text { metrics }\end{array}$ & Defect prediction \\
\hline BugHunter [51] & $\begin{array}{l}\text { Java source code; metrics; fix-inducing } \\
\text { commit; number of reported bugs }\end{array}$ & $\begin{array}{l}159 \mathrm{k} \text { pairs for } 3 \text { granularity levels } \\
\text { (file/class/method), } 15 \text { projects }\end{array}$ & $\begin{array}{l}\text { Analyzing the } \\
\text { importance of } \\
\text { complexity metrics }\end{array}$ \\
\hline $\begin{array}{l}\text { GitHub Bug } \\
\text { DataSet [52] }\end{array}$ & $\begin{array}{l}\text { Java source code; code metrics; } \\
\text { number of reported bugs and } \\
\text { vulnerabilities }\end{array}$ & 15 projects; $183 \mathrm{k}$ classes & Bug prediction \\
\hline $\begin{array}{l}\text { Unified Bug } \\
\text { Dataset [53] }\end{array}$ & $\begin{array}{l}\text { Java source code; code metrics; } \\
\text { number of reported bugs }\end{array}$ & 47,618 classes; 43,744 files & Bug prediction \\
\hline $\begin{array}{l}\text { Neural Code Translator } \\
\text { Dataset [54] }\end{array}$ & $\begin{array}{l}\text { Pairs of buggy and fixed abstracted } \\
\text { method-level fragments }\end{array}$ & $\begin{array}{l}46 \mathrm{k} \text { pairs of small fragments (under } 50 \text { tokens), } 50 \\
\mathrm{k} \text { pairs of medium fragments (under } 100 \text { tokens) }\end{array}$ & Code refinement \\
\hline BugsInPy [55] & $\begin{array}{l}\text { Pair of buggy and fixed Python } \\
\text { snippets, manually processed }\end{array}$ & 493 bugs from 17 projects & $\begin{array}{l}\text { Benchmark for testing } \\
\text { and debugging tools }\end{array}$ \\
\hline $\begin{array}{c}\text { Draper VDISC } \\
\text { Dataset [56] }\end{array}$ & $\begin{array}{l}\mathrm{C} \text { and } \mathrm{C}++ \text { source code, labeled for } \\
\text { potential vulnerabilities }\end{array}$ & $1.27 \mathrm{M}$ functions & Vulnerability Detection \\
\hline Refactory Dataset [57] & Python source code & 2442 correct and 1783 buggy program & Program repair \\
\hline Defect4J [58] & Java source code & 835 pairs of buggy and fixed fragments & $\begin{array}{l}\text { Software } \\
\text { testing research }\end{array}$ \\
\hline BugSwarm [59] & Java and Python source code & 3232 pairs of buggy and fixed fragments & $\begin{array}{l}\text { Software } \\
\text { testing research }\end{array}$ \\
\hline BuGL [60] & $\begin{array}{l}\mathrm{C}, \mathrm{C}++, \text { Java, and Python source code; } \\
\text { issues; pull requests }\end{array}$ & $\begin{array}{l}54 \text { projects; } 151 \text { k closed issues; } 10,187 \text { pull } \\
\text { requests }\end{array}$ & Bug localization \\
\hline Bugs.jar [61] & Java source code & 1158 pairs of buggy and and fixed fragments & Program repair \\
\hline
\end{tabular}

As with the other factors affecting the difficulty of constructing datasets, we can highlight that the distribution of the classes in the real code projects is often imbalanced. Usually, there are fewer buggy files or methods in a project than the correct ones. This may lead to the situation where the common classifiers would correctly detect the major class (correct code) and ignore the much smaller class of the defect-prone code. This will lead to bad performance of the model. 
To address this imbalance, several oversampling methods are proposed. In [62,63], the authors constructed hybrid approaches. It is based on the Synthetic Minority Over-Sampling Technique (SMOTE and SMOTUNED) for preparing the datasets and ensemble approaches for classifying the defective and correct code. In [22], the authors takes into account the proportion of the correct and defective code in each project in the dataset. To balance the classes, they duplicate the elements of the smaller class.

\subsection{Lack of Context}

Another problem is the complexity of the context for the code. Unlike the natural texts, the code element may depend on another element located far away, maybe, even in another code fragment. Moreover, it is often hard to say if the code element is defective without considering its context. If dataset consists of the pairs of bugged and fixed code fragments, it is often hard to extract the essence of defect.

Approaches based on the Transformer networks were aimed to NLP problems where data display a great deal of locality of reference. Most information about a token can be derived from its neighboring tokens [64]. Thus, most such models represent the source code as a sequence of tokens.

The traditional Transformer architectures based on self-attention matrices do not scale well because of quadratic complexity. Usually, they are designed to handle the input sequences with limited length (usually, 512 or 1024 tokens) [64,65]. Therefore, their applicability to understanding the context of the source code is limited.

There are several modifications to the Transformer architecture that improve its ability to comprehend long sequences [66-68]. These approaches alleviate the problem of limited length of the input, giving the Transformers the potential to work with a complex context of the source code.

Another approach is to capture the structural and global relations on the code, combining the sequence-based and graph-based models for code representation [34,69].

Thus, representing the code context is essential in the software defect prediction.

\section{RQ3. What Are the Trends in the Primary Studies on the Use of Deep Learning for the Software Defect Prediction?}

The earliest works, such as [16], utilize the deep learning techniques trying to extract the implicit features from the traditional explicit features (such as code metrics). The main drawback of this approach is that these traditional features usually cannot capture the semantic difference between the correct and defective code. Therefore, the combination of these features would also fail to do this [24].

Later approaches $[20,25]$ use the generic or tailored deep learning techniques to extract the semantic and syntactic features directly from the source code, usually, from the abstract syntax trees. These deep learned features are used in combination with the traditional ones in the machine classifiers to produce the accurate defect prediction.

Modern software development often prioritize writing the human-readable source code. This includes using the meaningful names for the functions and variables and writing the code documentation in natural language. This leads to a situation where we can extract the semantic information from the source code using the techniques originally intended for the NLP, such as the pre-trained language representations such as BERT [70].

Learning useful models with supervised setting is often difficult because labeled data are usually limited. Thus, many unsupervised approaches have been proposed recently to utilize the large unlabeled datasets that are more readily available. Usually, this means that pre-training is performed with automatic supervisions without manual annotation of the samples. Then, the model may be fine-tuned for the specific task using much smaller supervised data [31].

The most recent techniques in software engineering are based on using the generalpurposed pre-trained models for programming languages [34,71]. These models learn to "understand" the source code from unlabeled datasets using the self-supervised objectives. A large corpus of source code is used for pre-training. Usually, the objective is the Masked 
Language Modeling where at some positions the tokens are masked out and the model must predict the original token [32]. Utilizing these techniques alleviates the need for the task-specific architectures and training on large labeled datasets for each task separately.

\section{Conclusions}

One of the major challenges in modern software engineering is predicting defective code. Recent developments in the field of machine learning, especially the multi-layered neural networks and deep learning algorithms, provide powerful techniques, which utilize learning algorithms for representations of the source code that captures semantic and structural information.

This survey presents the latest research progress in software defect prediction using the deep learning techniques, such as the Transformer architectures. We formulate the main difficulties of the defect prediction problem as lack of data and complexity of context and discuss the ways to alleviate these problems.

Taking into account the latest trends in the machine learning techniques for the software defect prediction problem, we believe that progress in this field will be achieved largely due to the implementation of the following ideas.

- To reduce the requirements for the size of the labeled datasets, one should use the selfsupervised training on large corpora of the unlabeled data. In addition, it is necessary to use the unlabeled data for the pre-training of related tasks and to contribute to the fact that the trained models will have a deeper and more comprehensive understanding of the source code. This, in the turn, will allow one to find the deeper defects.

- To leverage the latest advances in the machine learning techniques in the natural language processing in the programming languages, we are already seeing the successful migration of these methods to solve various code understanding problems. For example, optimization of the self-attention mechanism for the transformers will allow one to use them for long sequences, which, in the turn, will lead to a more complete consideration of the code context for finding the defects.

- Often a defect is not limited to a single line of code or one function, and there are various ways to fix it. For example, a bug can be fixed either inside the function or at calling this function. Thus, the defect ceases to have specific coordinates inside the source file. In addition, not being an explicit defect, a line of code can become defective at a certain point in time. A changed context may lead to the fact that the purpose of the code changes, and, therefore, the old implementation no longer corresponds to the new requirements or specifications.

All this leads to a blurring of the concept of a defect. Thus, we come to the concepts of "potentially defective" code or "strange" code. In this regard, as promising problems, we want to note the task of finding an atypical (or anomalous) code and the task of the code refinement. These task require good representations of the code and code changes, taking into account the specifics of the source code, such as structure and context.

It is difficult to state which of the state-of-the-art models performs in the best way. There are no universally accepted standard benchmarks for the problem and different researchers utilize different performance metrics and use different data. Thus, the experimental results from the primary works cannot be directly compared. The existing comparative studies such as [72] show that while the state-of-the-art deep learning techniques usually perform better than standard deep learning and traditional metrics-based ones (achieving the increase of $\mathrm{F} 1$ from $60 \%$ up to $80 \%$ in some cases). None of the approaches achieves a consistently high performance in terms of recall, precision and accuracy sufficient for the practical application. Thus, the defect prediction problem remains an open one.

Author Contributions: Conceptualization, E.N.A., A.V.K.; Methodology, E.N.A., A.V.K., V.E.M.; Investigation, A.Yu.B., A.A.D., K.S.K., A.V.K., I.P.M., V.E.M.; Writing-original draft preparation, 
V.E.M., Writing-review and editing, E.N.A., K.S.K., A.V.K., V.E.M.; Supervision, E.N.A. All authors have read and agreed to the published version of the manuscript.

Funding: This research received no external funding.

Institutional Review Board Statement: Not applicable.

Informed Consent Statement: Not applicable.

Data Availability Statement: No new datasets were created in this survey. The datasets listed in Tables 1 and 2 are available from their respective authors.

Conflicts of Interest: The authors declare no conflict of interest.

\begin{tabular}{|c|c|}
\hline \multicolumn{2}{|c|}{ Abbreviations } \\
\hline & wing abbreviations are used \\
\hline AST & Abstract Syntax Tree \\
\hline CNN & Convolutional Neural Network \\
\hline DBN & Deep Belief Network \\
\hline DL & Deep Learning \\
\hline LSTM & Long Short Term Memory \\
\hline NLP & Natural Language Processing \\
\hline SDP & Software Defect Prediction \\
\hline
\end{tabular}

\section{References}

1. IEEE Standard Classification for Software Anomalies. IEEE Standard 1044-2009 (Revision of IEEE Standard 1044-1993). 2010, pp. 1-23. Available online: https:/ /ieeexplore.ieee.org/document/5399061 (accessed on 17 December 2020). [CrossRef]

2. Wang, S.; Liu, T.; Tan, L. Automatically Learning Semantic Features for Defect Prediction. In Proceedings of the 2016 IEEE/ACM 38th International Conference on Software Engineering (ICSE), Austin, TX, USA, 18-20 May 2016; pp. 297-308. [CrossRef]

3. Omri, S.; Sinz, C. Deep Learning for Software Defect Prediction: A Survey. In Proceedings of the IEEE/ACM 42nd International Conference on Software Engineering Workshops, ICSEW '20, Seoul, Korea, 6-11 July 2020; pp. 209-214. [CrossRef]

4. Yang, Y.; Xia, X.; Lo, D.; Grundy, J. A Survey on Deep Learning for Software Engineering. arXiv 2020, arXiv:cs.SE/2011.14597.

5. Shen, Z.; Chen, S. A Survey of Automatic Software Vulnerability Detection, Program Repair, and Defect Prediction Techniques. Secur. Commun. Netw. 2020, 2020, 8858010. [CrossRef]

6. Bryksin, T.; Petukhov, V.; Alexin, I.; Prikhodko, S.; Shpilman, A.; Kovalenko, V.; Povarov, N. Using Large-Scale Anomaly Detection on Code to Improve Kotlin Compiler. In Proceedings of the 17th International Conference on Mining Software Repositories, MSR '20, Seoul, Korea, 29-30 June 2020; pp. 455-465. [CrossRef]

7. Phan, A.V.; Le Nguyen, M. Convolutional neural networks on assembly code for predicting software defects. In Proceedings of the 2017 21st Asia Pacific Symposium on Intelligent and Evolutionary Systems (IES), Hanoi, Vietnam, 15-17 November 2017; pp. 37-42. [CrossRef]

8. Allamanis, M.; Barr, E.T.; Devanbu, P.; Sutton, C. A Survey of Machine Learning for Big Code and Naturalness. ACM Comput. Surv. 2018, 51. [CrossRef]

9. Chen, Z.; Monperrus, M. A Literature Study of Embeddings on Source Code. arXiv 2019, arXiv:cs.LG/1904.03061.

10. Sharmin, S.; Arefin, M.R.; Wadud, M.A.; Nower, N.; Shoyaib, M. SAL: An effective method for software defect prediction. In Proceedings of the 2015 18th International Conference on Computer and Information Technology (ICCIT), Dhaka, Bangladesh, 21-23 December 2015; pp. 184-189. [CrossRef]

11. Dam, H.K.; Tran, T.; Pham, T.; Ng, S.W.; Grundy, J.; Ghose, A. Automatic Feature Learning for Predicting Vulnerable Software Components. IEEE Trans. Softw. Eng. 2018, 47, 67-85. [CrossRef]

12. Mikolov, T.; Chen, K.; Corrado, G.; Dean, J. Efficient Estimation of Word Representations in Vector Space. arXiv 2013, arXiv:cs.CL/1301.3781.

13. Zhang, J.; Wang, X.; Zhang, H.; Sun, H.; Wang, K.; Liu, X. A Novel Neural Source Code Representation Based on Abstract Syntax Tree. In Proceedings of the 2019 IEEE/ACM 41st International Conference on Software Engineering (ICSE), Montreal, QC, Canada, 25-31 May 2019; pp. 783-794. [CrossRef]

14. Pradel, M.; Sen, K. DeepBugs: A Learning Approach to Name-Based Bug Detection. Proc. ACM Program. Lang. 2018, 2. [CrossRef]

15. Bengio, Y. Learning Deep Architectures for AI. Found. Trends Mach. Learn. 2009, 2, 1-127. [CrossRef]

16. Yang, X.; Lo, D.; Xia, X.; Zhang, Y.; Sun, J. Deep Learning for Just-in-Time Defect Prediction. In Proceedings of the 2015 IEEE International Conference on Software Quality, Reliability and Security, Vancouver, BC, Canada, 3-5 August 2015; pp. 17-26. [CrossRef]

17. Yang, X.; Lo, D.; Xia, X.; Sun, J. TLEL: A two-layer ensemble learning approach for just-in-time defect prediction. Inf. Softw. Technol. 2017, 87, 206-220. [CrossRef] 
18. Wang, S.; Liu, T.; Nam, J.; Tan, L. Deep Semantic Feature Learning for Software Defect Prediction. IEEE Trans. Softw. Eng. 2018, 46, 1267-1293. [CrossRef]

19. Hochreiter, S.; Schmidhuber, J. Long Short-Term Memory. Neural Comput. 1997, 9, 1735-1780. [CrossRef]

20. Dam, H.K.; Pham, T.; Ng, S.W.; Tran, T.; Grundy, J.; Ghose, A.; Kim, T.; Kim, C.J. Lessons Learned from Using a Deep Tree-Based Model for Software Defect Prediction in Practice. In Proceedings of the 2019 IEEE/ ACM 16th International Conference on Mining Software Repositories (MSR), Montreal, QC, Canada, 25-31 May 2019; pp. 46-57. [CrossRef]

21. Habib, A.; Pradel, M. Neural Bug Finding: A Study of Opportunities and Challenges. arXiv 2019, arXiv:cs.SE/1906.00307.

22. Shi, K.; Lu, Y.; Chang, J.; Wei, Z. PathPair2Vec: An AST path pair-based code representation method for defect prediction. J. Comput. Lang. 2020, 59, 100979. [CrossRef]

23. Goodfellow, I.; Bengio, Y.; Courville, A. Deep Learning; MIT Press: Cambridge, MA, USA, 2016. Available online: http: / / www.deeplearningbook.org (accessed on 17 December 2020).

24. Li, J.; He, P.; Zhu, J.; Lyu, M.R. Software Defect Prediction via Convolutional Neural Network. In Proceedings of the 2017 IEEE International Conference on Software Quality, Reliability and Security (QRS), Prague, Czech Republic, 25-29 July 2017; pp. 318-328. [CrossRef]

25. Hoang, T.; Khanh Dam, H.; Kamei, Y.; Lo, D.; Ubayashi, N. DeepJIT: An End-to-End Deep Learning Framework for Just-in-Time Defect Prediction. In Proceedings of the 2019 IEEE/ACM 16th International Conference on Mining Software Repositories (MSR), Montreal, QC, Canada, 25-31 May 2019, pp. 34-45. [CrossRef]

26. Xu, Z.; Li, S.; Xu, J.; Liu, J.; Luo, X.; Zhang, Y.; Zhang, T.; Keung, J.; Tang, Y. LDFR: Learning deep feature representation for software defect prediction. J. Syst. Softw. 2019, 158, 110402. [CrossRef]

27. Qiu, S.; Lu, L.; Cai, Z.; Jiang, S. Cross-Project Defect Prediction via Transferable Deep Learning-Generated and Handcrafted Features. In Proceedings of the 31st International Conference on Software Engineering \& Knowledge Engineering (SEKE 2019), Lisbon, Portugal, 10-12 July 2019; pp. 1-6. Available online: http://ksiresearch.org/seke/seke19paper/seke19paper_70.pdf (accessed on 17 December 2020).

28. Cai, Z.; Lu, L.; Qiu, S. An Abstract Syntax Tree Encoding Method for Cross-Project Defect Prediction. IEEE Access 2019, 7, 170844-170853. [CrossRef]

29. Liu, Y.; Ott, M.; Goyal, N.; Du, J.; Joshi, M.; Chen, D.; Levy, O.; Lewis, M.; Zettlemoyer, L.; Stoyanov, V. RoBERTa: A Robustly Optimized BERT Pretraining Approach. arXiv 2019, arXiv:cs.CL/1907.11692.

30. Vaswani, A.; Shazeer, N.; Parmar, N.; Uszkoreit, J.; Jones, L.; Gomez, A.N.; Kaiser, L.; Polosukhin, I. Attention Is All You Need. arXiv 2017, arXiv:cs.CL/1706.03762.

31. Kanade, A.; Maniatis, P.; Balakrishnan, G.; Shi, K. Learning and Evaluating Contextual Embedding of Source Code. In Proceedings of the 37th International Conference on Machine Learning; Daumé , H., III, Singh, A., Eds.; PMLR: 2020; Volume 119, pp. 5110-5121. Available online: http:/ / proceedings.mlr.press/v119/kanade20a.html (accessed on 17 December 2020).

32. Feng, Z.; Guo, D.; Tang, D.; Duan, N.; Feng, X.; Gong, M.; Shou, L.; Qin, B.; Liu, T.; Jiang, D.; et al. CodeBERT: A Pre-Trained Model for Programming and Natural Languages. arXiv 2020, arXiv:cs.CL/2002.08155.

33. Humphreys, J.; Dam, H.K. An Explainable Deep Model for Defect Prediction. In Proceedings of the 22019 IEEE/ACM 7th International Workshop on Realizing Artificial Intelligence Synergies in Software Engineering (RAISE), Montreal, QC, Canada, 28 May 2019; pp. 49-55. [CrossRef]

34. Guo, D.; Ren, S.; Lu, S.; Feng, Z.; Tang, D.; Liu, S.; Zhou, L.; Duan, N.; Svyatkovskiy, A.; Fu, S.; et al. GraphCodeBERT: Pre-training Code Representations with Data Flow. arXiv 2021, arXiv:cs.SE/2009.08366.

35. Tong, H.; Liu, B.; Wang, S. Software defect prediction using stacked denoising autoencoders and two-stage ensemble learning. Inf. Softw. Technol. 2018, 96, 94-111. [CrossRef]

36. Tran, H.D.; Hanh, L.T.M.; Binh, N.T. Combining feature selection, feature learning and ensemble learning for software fault prediction. In Proceedings of the 2019 11th International Conference on Knowledge and Systems Engineering (KSE), Da Nang, Vietnam, 24-26 October 2019; pp. 1-8. [CrossRef]

37. Zhao, L.; Shang, Z.; Zhao, L.; Zhang, T.; Tang, Y.Y. Software defect prediction via cost-sensitive Siamese parallel fully-connected neural networks. Neurocomputing 2019, 352, 64-74. [CrossRef]

38. Qiu, Y.; Liu, Y.; Liu, A.; Zhu, J.; Xu, J. Automatic Feature Exploration and an Application in Defect Prediction. IEEE Access 2019, 7, 112097-112112. [CrossRef]

39. Zhou, T.; Sun, X.; Xia, X.; Li, B.; Chen, X. Improving defect prediction with deep forest. Inf. Softw. Technol. 2019, 114, 204-216. [CrossRef]

40. Xu, J.; Wang, F.; Ai, J. Defect Prediction With Semantics and Context Features of Codes Based on Graph Representation Learning. IEEE Trans. Reliab. 2020, 1-13. [CrossRef]

41. Raychev, V.; Bielik, P.; Vechev, M. Probabilistic Model for Code with Decision Trees. SIGPLAN Not. 2016, 51, 731-747. [CrossRef]

42. Raychev, V.; Bielik, P.; Vechev, M.; Krause, A. Learning Programs from Noisy Data. SIGPLAN Not. 2016, 51, 761-774. [CrossRef]

43. Alon, U.; Brody, S.; Levy, O.; Yahav, E. code2seq: Generating Sequences from Structured Representations of Code. arXiv 2019, arXiv:cs.LG/1808.01400.

44. Allamanis, M.; Sutton, C. Mining source code repositories at massive scale using language modeling. In Proceedings of the 2013 10th Working Conference on Mining Software Repositories (MSR), San Francisco, CA, USA, 18-19 May 2013; pp. 207-216. [CrossRef] 
45. Iyer, S.; Konstas, I.; Cheung, A.; Zettlemoyer, L. Summarizing source code using a neural attention model. In Proceedings of the 54th Annual Meeting of the Association for Computational Linguistics (Volume 1: Long Papers), Berlin, Germany, 7-12 August 2016; pp. 2073-2083.

46. Allamanis, M.; Brockschmidt, M.; Khademi, M. Learning to Represent Programs with Graphs. arXiv 2018, arXiv:cs.LG/1711.00740.

47. Mauša, G.; Galinac-Grbac, T.; Dalbelo-Bašić, B. A systematic data collection procedure for software defect prediction. Comput. Sci. Inf. Syst. 2016, 13, 173-197. [CrossRef]

48. Sayyad Shirabad, J.; Menzies, T. The PROMISE Repository of Software Engineering Databases; School of Information Technology and Engineering, University of Ottawa: Ottawa, ON, Canada, 2005. Available online: http:/ / promise.site.uottawa.ca/SERepository/ (accessed on 17 December 2020).

49. Shepperd, M.; Song, Q.; Sun, Z.; Mair, C. NASA MDP Software Defects Data Sets. 2018. Available online: https://figshare.com/ collections/NASA_MDP_Software_Defects_Data_Sets/4054940/1 (accessed on 17 December 2020).

50. Afric, P.; Sikic, L.; Kurdija, A.S.; Silic, M. REPD: Source code defect prediction as anomaly detection. J. Syst. Softw. 2020, 168, 110641. [CrossRef]

51. Ferenc, R.; Gyimesi, P.; Gyimesi, G.; Tóth, Z.; Gyimóthy, T. An automatically created novel bug dataset and its validation in bug prediction. J. Syst. Softw. 2020, 169, 110691. [CrossRef]

52. Tóth, Z.; Gyimesi, P.; Ferenc, R. A Public Bug Database of GitHub Projects and Its Application in Bug Prediction. In Proceedings of the Computational Science and Its Applications-ICCSA, Beijing, China, 4-7 July 2016; Springer International Publishing: Cham, Switzerland, 2016; pp. 625-638. [CrossRef]

53. Ferenc, R.; Tóth, Z.; Ladányi, G.; Siket, I.; Gyimóthy, T. A public unified bug dataset for java and its assessment regarding metrics and bug prediction. Softw. Qual. J. 2020, 28, 1447-1506. [CrossRef]

54. Tufano, M.; Watson, C.; Bavota, G.; Penta, M.D.; White, M.; Poshyvanyk, D. An Empirical Study on Learning Bug-Fixing Patches in the Wild via Neural Machine Translation. ACM Trans. Softw. Eng. Methodol. 2019, 28, 1-29. [CrossRef]

55. Widyasari, R.; Sim, S.Q.; Lok, C.; Qi, H.; Phan, J.; Tay, Q.; Tan, C.; Wee, F.; Tan, J.E.; Yieh, Y.; et al. BugsInPy: A database of existing bugs in Python programs to enable controlled testing and debugging studies. In Proceedings of the ESEC/FSE '20: 28th ACM Joint European Software Engineering Conference and Symposium on the Foundations of Software Engineering, Virtual Event, USA, 8-13 November 2020; Devanbu, P., Cohen, M.B., Zimmermann, T., Eds.; ACM: New York, NY, USA, 2020; pp. 1556-1560. [CrossRef]

56. Russell, R.; Kim, L.; Hamilton, L.; Lazovich, T.; Harer, J.; Ozdemir, O.; Ellingwood, P.; McConley, M. Automated Vulnerability Detection in Source Code Using Deep Representation Learning. In Proceedings of the 2018 17th IEEE International Conference on Machine Learning and Applications (ICMLA), Orlando, FL, USA, 17-20 December 2018; pp. 757-762. [CrossRef]

57. Hu, Y.; Ahmed, U.Z.; Mechtaev, S.; Leong, B.; Roychoudhury, A. Re-factoring based Program Repair applied to Programming Assignments. In Proceedings of the 2019 34th IEEE/ACM International Conference on Automated Software Engineering (ASE), IEEE/ACM, San Diego, CA, USA, 11-15 November 2019; pp. 388-398. [CrossRef]

58. Just, R.; Jalali, D.; Ernst, M.D. Defects4J: A database of existing faults to enable controlled testing studies for Java programs. In Proceedings of the 2014 International Symposium on Software Testing and Analysis, San Jose, CA, USA, 21-25 July 2014; pp. 437-440. [CrossRef]

59. Tomassi, D.A.; Dmeiri, N.; Wang, Y.; Bhowmick, A.; Liu, Y.; Devanbu, P.T.; Vasilescu, B.; Rubio-González, C. BugSwarm: Mining and Continuously Growing a Dataset of Reproducible Failures and Fixes; ICSE.IEEE/ACM: Montreal, QC, Canada, 2019; pp. 339-349. [CrossRef]

60. Muvva, S.; Rao, A.E.; Chimalakonda, S. BuGL-A Cross-Language Dataset for Bug Localization. arXiv 2020, arXiv:cs.SE/2004.08846.

61. Saha, R.K.; Lyu, Y.; Lam, W.; Yoshida, H.; Prasad, M.R. Bugs.Jar: A Large-Scale, Diverse Dataset of Real-World Java Bugs. In Proceedings of the 15th International Conference on Mining Software Repositories (MSR '18), Gothenburg, Sweden, 28-29 May 2018; Association for Computing Machinery: New York, NY, USA, 2018; pp. 10-13. [CrossRef]

62. Alsawalqah, H.; Faris, H.; Aljarah, I.; Alnemer, L.; Alhindawi, N. Hybrid SMOTE-Ensemble Approach for Software Defect Prediction. In Software Engineering Trends and Techniques in Intelligent Systems; Silhavy, R., Silhavy, P., Prokopova, Z., Senkerik, R., Kominkova Oplatkova, Z., Eds.; Springer International Publishing: Cham, Switzerland, 2017; pp. 355-366.

63. Agrawal, A.; Menzies, T. Is "Better Data" Better than "Better Data Miners"? On the Benefits of Tuning SMOTE for Defect Prediction. In Proceedings of the 40th International Conference on Software Engineering (ICSE '18), Gothenburg, Sweden, 27 May-3 June 2018; Association for Computing Machinery: New York, NY, USA, 2018; pp. 1050-1061, [CrossRef]

64. Tay, Y.; Dehghani, M.; Bahri, D.; Metzler, D. Efficient Transformers: A Survey. arXiv 2020, arXiv:cs.LG/2009.06732.

65. Tay, Y.; Dehghani, M.; Abnar, S.; Shen, Y.; Bahri, D.; Pham, P.; Rao, J.; Yang, L.; Ruder, S.; Metzler, D. Long Range Arena: A Benchmark for Efficient Transformers. arXiv 2020, arXiv:cs.LG/2011.04006.

66. Zaheer, M.; Guruganesh, G.; Dubey, A.; Ainslie, J.; Alberti, C.; Ontanon, S.; Pham, P.; Ravula, A.; Wang, Q.; Yang, L.; et al. Big Bird: Transformers for Longer Sequences. arXiv 2021, arXiv:cs.LG/2007.14062.

67. Fiok, K.; Karwowski, W.; Gutierrez, E.; Davahli, M.R.; Wilamowski, M.; Ahram, T.; Al-Juaid, A.; Zurada, J. Text Guide: Improving the quality of long text classification by a text selection method based on feature importance. arXiv 2021, arXiv:cs.AI/2104.07225.

68. Beltagy, I.; Peters, M.E.; Cohan, A. Longformer: The Long-Document Transformer. arXiv 2020, arXiv:cs.CL/2004.05150.

69. Hellendoorn, V.J.; Sutton, C.; Singh, R.; Maniatis, P.; Bieber, D. Global Relational Models of Source Code. In Proceedings of the International Conference on Learning Representations (ICLR), Virtual Event, 26 April-1 May 2020. 
70. Devlin, J.; Chang, M.W.; Lee, K.; Toutanova, K. BERT: Pre-training of Deep Bidirectional Transformers for Language Understanding. In Proceedings of the 2019 Conference of the North American Chapter of the Association for Computational Linguistics: Human Language Technologies, Volume 1 (Long and Short Papers), Minneapolis, MN, USA, 2-7 June, 2019; Association for Computational Linguistics: Minneapolis, MN, USA, 2019; pp. 4171-4186. [CrossRef]

71. Karampatsis, R.M.; Sutton, C. SCELMo: Source Code Embeddings from Language Models. arXiv 2020, arXiv:cs.SE/2004.13214.

72. Herbold, S.; Trautsch, A.; Grabowski, J. A Comparative Study to Benchmark Cross-Project Defect Prediction Approaches. IEEE Trans. Softw. Eng. 2018, 44, 811-833. [CrossRef] 